\title{
064 SLEEP AND MEMORY CONSOLIDATION IN TEA
}

Kathryn Atherton, ${ }^{1}$ Adam Zeman, ${ }^{2}$ Kia Nobre, ${ }^{1}$ Christopher Butler ${ }^{1} .{ }^{1}$ University of Oxford; '2University of Exeter

\subsection{6/jnnp-2014-309236.64}

Accelerated long-term forgetting (ALF), which is common in transient epileptic amnesia (TEA), is a form of memory impairment in which learning and initial retention of information appear normal but subsequent forgetting is excessively rapid. A deficit in sleep-dependent memory consolidation has been proposed as an explanation for ALF. If this proposal were correct, then sleep would not benefit memory retention in people with ALF as much as in healthy people, and ALF might only be apparent when the retention interval contains sleep. To test this theory, we compared memory retention over a night of sleep and a day of wakefulness. We found, contrary to the hypothesis, that sleep benefits memory retention in TEA patients with ALF and that this benefit is no smaller in magnitude than that seen in healthy controls. Indeed, patients performed significantly more poorly than the controls only in the wake condition and not the sleep condition. These results indicate that ALF is not caused by disruption of sleep-dependent memory consolidation. Instead, ALF may be due to an encoding abnormality that goes undetected on behavioural assessments of learning, or by a deficit in memory consolidation processes that are not sleep-dependent. 